\title{
Identification of materials with magnetic characteristics by neural networks
}

\author{
Sedat Nazlibilek ${ }^{\mathrm{a}, \mathrm{b}}$, Yavuz Ege ${ }^{\mathrm{c}, *}$, Osman Kalender ${ }^{\mathrm{d}}$, Mehmet Gökhan Sensoy ${ }^{\mathrm{e}}$, Deniz Karacor ${ }^{\mathrm{f}}$, \\ Murat Hüsnü Sazlı ${ }^{\mathrm{f}}$ \\ ${ }^{a}$ Bilkent University, Nanotechnology Research Center (Nanotam), 06800 Ankara, Turkey \\ ${ }^{\mathrm{b}}$ Atilim University, Faculty of Engineering, Department of Mechatronics Engineering, 06800 Ankara, Turkey \\ ${ }^{\mathrm{c}}$ Balikesir University, Necatibey Faculty of Education, Department of Physics, 10100 Balikesir, Turkey \\ ${ }^{\mathrm{d}}$ Turkish Military College, Department of Technical Sciences, 06100 Bakanliklar, Ankara, Turkey \\ ${ }^{\mathrm{e}}$ Middle East Technical University, Faculty of Arts and Sciences, Department of Physics, 06800 Ankara, Turkey \\ ${ }^{\mathrm{f}}$ Ankara Üniversity, Faculty of Engineering, Electronics Engineering Department, 06100 Ankara, Turkey
}

\section{A R T I C L E I N F O}

\section{Article history:}

Received 18 August 2011

Received in revised form 10 November 2011

Accepted 28 December 2011

Available online 11 January 2012

\section{Keywords:}

Anisotropic magnetoresistive sensor (AMR)

Magnetic anomaly

Magnetic materials

Remote sensing

Neural networks

\begin{abstract}
A B S T R A C T
In industry, there is a need for remote sensing and autonomous method for the identification of the ferromagnetic materials used. The system is desired to have the characteristics of improved accuracy and low power consumption. It must also autonomous and fast enough for the decision. In this work, the details of inaccurate and low power remote sensing mechanism and autonomous identification system are given. The remote sensing mechanism utilizes KMZ51 anisotropic magneto-resistive sensor with high sensitivity and low power consumption. The images and most appropriate mathematical curves and formulas for the magnetic anomalies created by the magnetic materials are obtained by 2-D motion of the sensor over the material. The contribution of the paper is the use of the images obtained by the measurement of the perpendicular component of the Earth magnetic field that is a new method for the purpose of identification of an unknown magnetic material. The identification system is based on two kinds of neural network structures. The MultiLayer Perceptron (MLP) and the Radial Basis Function (RBF) network types are used for training of the neural networks. In this work, 23 different materials such as SAE/AISI 1030, 1035, 1040, 1060, 4140 and 8260 are identified. Besides the ferromagnetic materials, three objects are also successfully identified. Two of them are anti-personal and anti-tank mines and one is an empty can box. It is shown that the identification system can also be used as a buried mine identification system. The neural networks are trained with images which are originally obtained by the remote sensing system and the system is operated by images with added Gaussian white noises.
\end{abstract}

Crown Copyright @ 2012 Published by Elsevier Ltd. All rights reserved.

\section{Introduction}

In recent years, a lot of intelligent systems and algorithms such as neural networks, fuzzy systems, belief functions, and learning and/or training algorithms have been developed and applied for the identification of a variety of quantities successfully. Also, today, a variety of methods are used for remotely sensing the objects and materials.

\footnotetext{
* Corresponding author.

E-mail address: yavuzege@gmail.com (Y. Ege).
}

They have a spectrum of applications varying from acoustics to all kind of imaging including $\mathrm{THz}$ band. In this work, a remote sensing and identification of the dimensions and magnetic characteristics of materials which are widely used in industry by use of neural networks (NNs) is developed, implemented and comprehensive experiments are carried out. This work is the continuation of our previous study [1]. In the works, we have concentrated on the detection of improved and manufacturing-type steels with ferromagnetic characteristics widely used in industrial applications. The remote sensing is achieved by using a 
magnetic anomaly method [2-9]. The neural networks are extensively used for identification and classification purposes $[10,11]$.

Since the magnetic permeability of this type of materials is very high, they attract the magnetic field lines of the Earth which are parallel to the ground toward themselves. In this case, there will be two components of the lines: One is horizontal, and the other is vertical. If the vertical component that occurred with the availability of magnetic material can be sensed by the magnetic sensor, then it will be possible to identify the material. In order to achieve it, the sensitivity of the sensor becomes important. For this reason, in this paper, a KMZ51 magnetosensitive sensor with low power and high sensitivity is used. The study presented here and the previous paper [1] is unique since reduced power consumption and highly accurate measurements can be achieved. Furthermore, this is a new approach for the identification of material with magnetic characteristics which can be achieved based on the detection of the vertical component of the Earth's magnetic field [5-9]. In addition, if the field lines of the Earth at a location where magnetic materials are found are constant and homogeneous, then it may give rise to some difficulties in identifying the material. This work has been carried out in an environment where the Earth magnetic field is homogeneous and the value of it is $4.4 \times 10^{-5} \mathrm{~T}$. After installing the experiment measurement system in this environment, some materials made up of steel with various chemical compositions and magnetic permeabilities have been brought to the scanning area of the sensor. The data obtained by scanning a plane which is parallel to the plane of the materials have been analyzed and used to capture some geometric properties of the materials. Now, we introduce an intelligent subsystem, a neural network (Fig. 1), to our sensing mechanism for autonomous and fast identification. In industry, there is a need for remote sensing and autonomous method for the identification of the ferromagnetic materials used. The system is desired to have the characteristics of improved accuracy
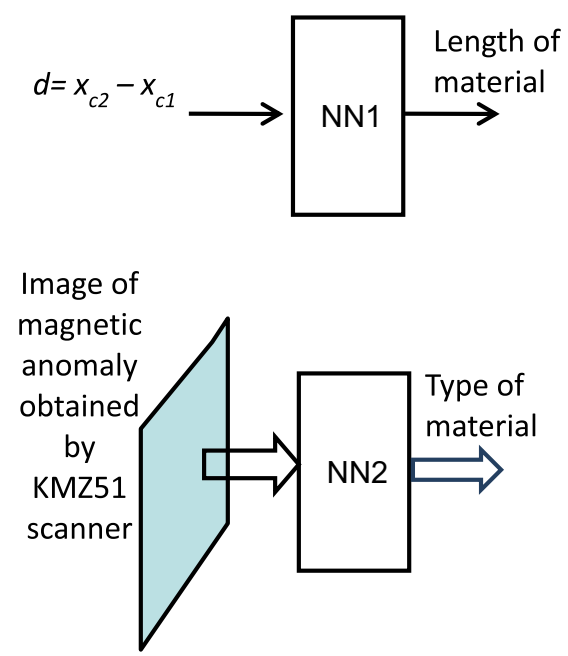

Fig. 1. The general diagram of the neural network (NN) system used in the identification process. and low power consumption. It must also autonomous and fast enough for the decision. In this work, the details of an accurate and low power remote sensing mechanism and autonomous identification system are given.

The contribution of the paper is the use of the images obtained by the measurement of the perpendicular component of the Earth magnetic field that is a new method for the purpose of identification of an unknown magnetic material. The identification system is based on two kinds of neural network structures. The MultiLayer Perceptron (MLP) and the Radial Basis Function (RBF) network types are used for training of the neural networks. In this work, 23 different materials such as SAE/AISI 1030, 1035, 1040, 1060, 4140 and 8260 are identified. Besides the ferromagnetic materials, three objects are also successfully identified. Two of them are anti-personal and anti-tank mines and one is an empty can box. It is shown that the identification system can also be used as a buried mine identification system. The neural networks are trained with images which are originally obtained by the remote sensing system and the system is operated by images with added Gaussian white noises.

The paper is organized as follows. Section 2 describes the measurement system. In Section 3, the structure of the identification system is given. Section 4 presents the operation of the identification system and experimental results.

\section{Measurement system}

A scanner moving in three dimensions with a KMZ51 magneto-resistive sensor is used to measure the magnetic anomaly created by the magnetic material whose magnetic characteristics is to be identified (Fig. 2) [1-12]. The analog data (voltage) produced by the sensor is digitized by a 24-bit ADC (AD7714) and transferred to the computer for further processing (Fig. 3). $z$-Component of the anomaly is measured.

The materials used in this study are listed in Table 1.

The data collected to create the image of magnetic anomalies are the strengths of the magnetic field at the

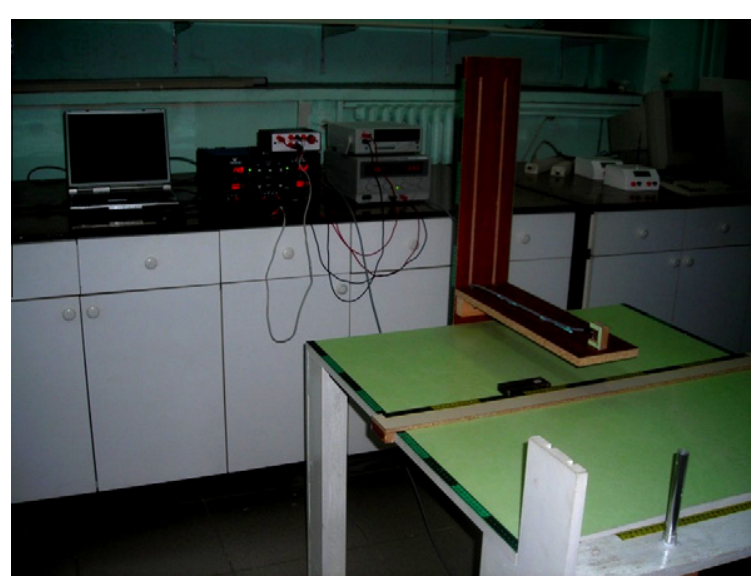

Fig. 2. The 3D scanner used for data acquisition. 


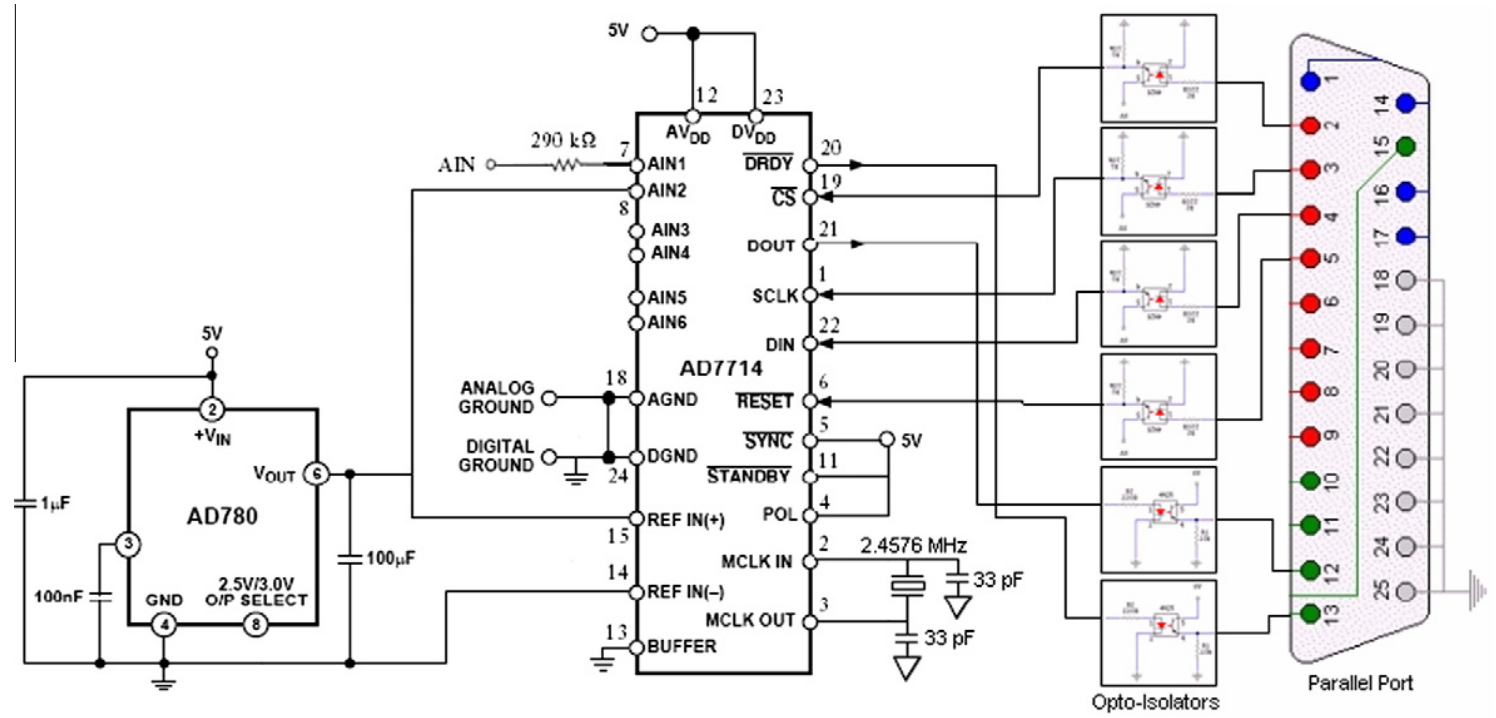

Fig. 3. The 24-bit data acquisition circuit.

Table 1

The chemical contents of the magnetic materials.

\begin{tabular}{llllllll}
\hline SAE/AISI & $\mathrm{C}$ & $\mathrm{Si}$ & $\mathrm{Mn}$ & $P_{\max }$ & $S_{\max }$ & $\mathrm{Cr}$ & $\mathrm{Mo}$ \\
\hline \multirow{2}{*}{1030} & 0.28 & 0.15 & 0.60 & 0.040 & 0.050 & - & - \\
& 0.34 & 0.35 & 0.90 & & & & \\
1035 & 0.32 & 0.15 & 0.60 & 0.040 & 0.050 & - & - \\
& 0.38 & 0.35 & 0.90 & & & & \\
1040 & 0.37 & 0.15 & 0.60 & 0.040 & 0.050 & - & - \\
& 0.44 & 0.35 & 0.90 & & & & \\
1060 & 0.55 & 0.15 & 0.60 & 0.040 & 0.050 & - & - \\
& 0.65 & 0.35 & 0.90 & & & & \\
4140 & 0.38 & 0.15 & 0.50 & 0.035 & 0.035 & 0.90 & 0.15 \\
& 0.45 & 0.40 & 0.80 & & & 1.20 & 0.30 \\
8620 & 0.18 & 0.15 & 0.60 & 0.040 & 0.040 & 0.40 & 0.15 \\
& 0.23 & 0.35 & 0.90 & & & 0.60 & 0.25 \\
\hline
\end{tabular}

point where the measurement is carried out. They are put into a matrix $\mathbf{M}_{\mathrm{i}}$ The elements of the matrix $\mathbf{M}_{\mathrm{i}}$ are listed as a $k$ dimensional vector, $\mathbf{m}$, where $k=m \times n, m$ is the number of elements in the row and $n$ is the number of elements in the column of the matrix $\mathbf{M}_{\mathrm{i}}$. This vector is used as the input to the neural network used. The input vector can be written as

$\mathbf{m}=\left[m_{1}, m_{2}, m_{3}, \ldots, m_{k}\right]^{T}$

The variation of the sensor output voltage about at the center of the edge on the $y$ axis and through the $x$-axis and the curve fitted on it are shown in Fig. 4. The mathematical formula for the Gaussian curve fitted is

$$
\begin{aligned}
V_{\text {output }}= & V_{0}+\frac{A_{1}}{W_{1} \times \sqrt{\frac{\pi}{2}}} \times e^{-2\left(\frac{x-x_{c 1}}{W_{1}}\right)^{2}}+\frac{A_{2}}{W_{2} \times \sqrt{\frac{\pi}{2}}} \\
& \times e^{-2\left(\frac{x-x_{c 2}}{W_{2}}\right)^{2}}
\end{aligned}
$$

where $V_{0}$; constant value from the sensor when there is no material; $x_{c 1}$ and $x_{\mathrm{c} 2}$; the $x$ coordinates of two peaks of the

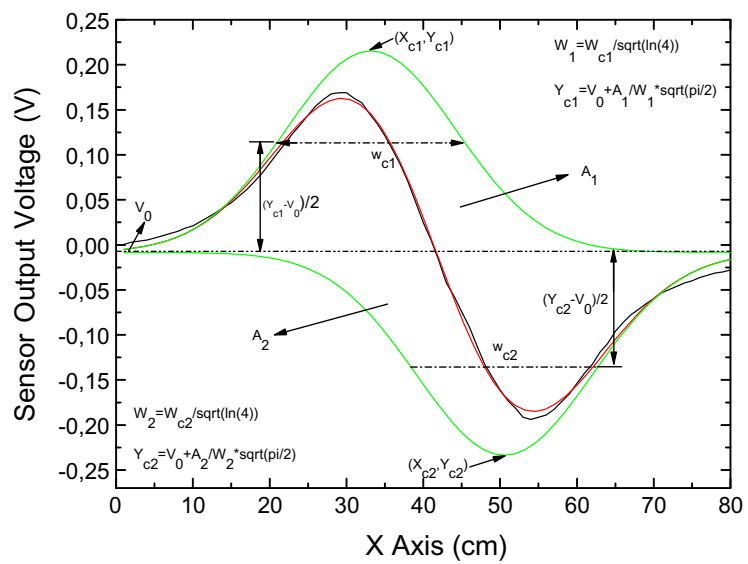

Fig. 4. Sensor output voltage variations through $x$-axis (a) measured curve; (b) fitted Gaussian curves.

Gaussian curves; $W_{1}$ and $W_{2}$; widths of the Gaussian curves; $A_{1}$ and $A_{2}$; the area between the curves and the $V_{0}$ asymptote. Although the sensor output voltage characteristics are similar in shape for the materials, the values of the parameters of the curves $V_{0}, x_{\mathrm{c} 1}, W_{1}, A_{1}, x_{\mathrm{c} 2}, W_{2}, A_{2}$ may change. The identification process actually is based on these variables and parameters for different materials. However, in this work only the images obtained are trained and identified by neural nets.

The length or dimension of a material can be determined from the difference between the $x$ coordinates of the peak values of the Gaussian curves. Therefore, a database composed of the curves of $d=x_{c 2}-x_{c 1}$ versus length for each type of material has to be created. Table 2 gives an example of such a database. These curves are used to train the dimension classifier, $\mathrm{NN}_{1}$ which in turn is used to obtain the dimension of the material under investigation. 
Table 2

A database composed of the curves of $D=X_{C 2}-X_{C 1}$ versus length, $l$, for each type of material.

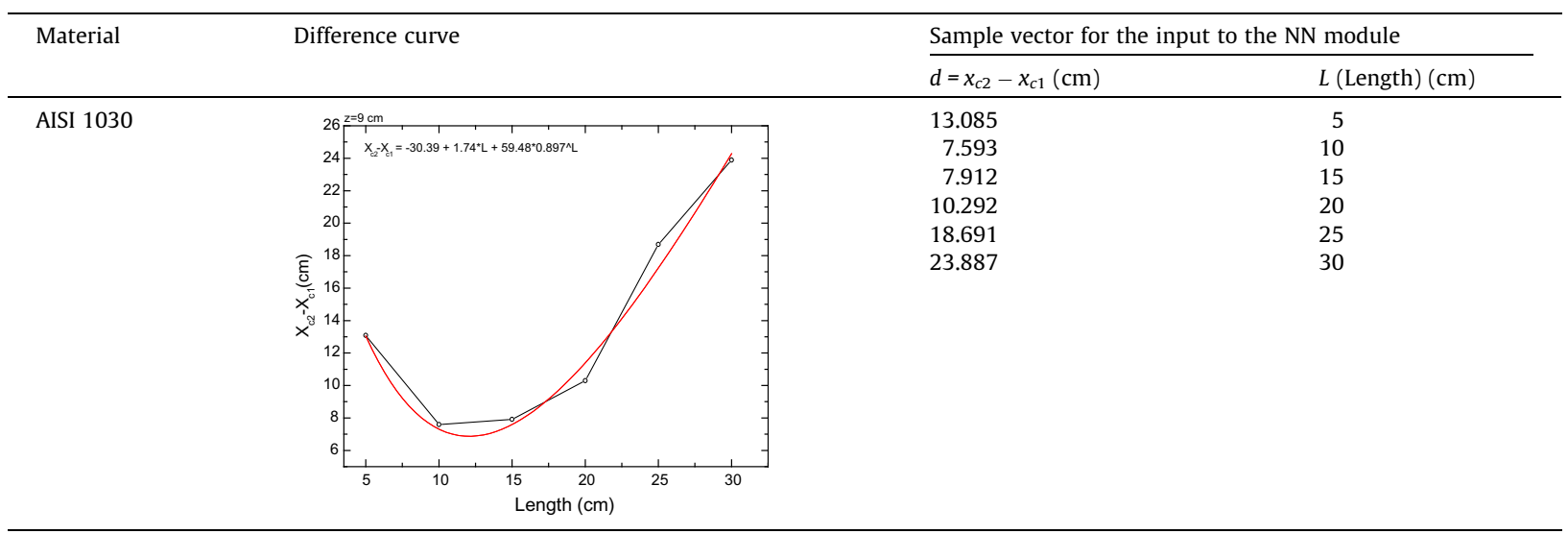

Similarly, the curves of the sensor output voltage level versus height of the sensor which can be used for the determination of the type of the materials can be seen in Table 4 . The curves are also sampled and the sampled values are listed. These curves can be used to train another neural network module identifying the type of the material. However, the use of these curves are optional in this study, and only the images are enough for material identification.

The neural network (NN) system used for the identification process is shown in Fig. 1. The NN consists of two modules. The first module, $\mathrm{NN}_{1}$, uses the difference value between the two peaks of the Gaussian curve as the input and the length of the material as the output. It identifies the dimension of the material. It is called the "dimension classifier". The second module, $\mathrm{NN}_{2}$, has the images of the magnetic anomaly as the input vector and the type code of the material as the output. It identifies the type of the material. It is called the "image classifier". This module also accepts the inputs from the other modules to fortify the decision for the identification. It is the main classifier of the overall system.

The neural network modules, NNs, transform the input vectors into outputs that can be used as the information of identification of materials. These transformations can be written as follows:

$L=\left(\boldsymbol{T}_{\mathrm{NN} 1}\right) \cdot d$

Table 3

The codes of the type of magnetic materials.

\begin{tabular}{lllr}
\hline Material & Code & \multicolumn{2}{c}{ Relative permeability } \\
\cline { 3 - 4 } & & $\mu_{x}$ & $\mu_{z}$ \\
\hline AISI 1030 & 1 & 1560 & 350 \\
AISI 1035 & 2 & 1650 & 405 \\
AISI 1040 & 3 & 235 & 2920 \\
AISI 1060 & 4 & 295 & 3570 \\
AISI 4140 & 5 & 3220 & 220 \\
AISI 8620 & 6 & 1520 & 305 \\
\hline
\end{tabular}

$\boldsymbol{T}_{\boldsymbol{m}}=\left(\boldsymbol{T}_{N N 2}\right) \cdot \boldsymbol{m}$

where $L$ is the length of the material in $\mathrm{cm} ; \boldsymbol{T}_{\boldsymbol{m}}$ is a vector whose entries $\in\{1,-1\}$ : the type code of the material.

As seen, the length of the material is directly produced from the $\mathrm{NN}$ in $\mathrm{cm}$. The type of the material is coded as an integer number as seen in Table 3.

\subsection{Training period}

During the training period, the data in Tables 2 and 3 in the form of vectors described as in the above are applied to the neural network modules. After the training period, the weights are adjusted to give appropriate outputs based on the inputs applied.

\subsection{Transformation period}

The networks are operated to transform a measured data into information that it identifies the dimension and the type of the magnetic material. During the transformation, the input data obtained by the sensor scanner system and transferred to the computer is applied to the inputs of the neural network modules the output is obtained immediately. The neural network system produces the identified output. Several examples are shown in Table 4.

\section{The structure of the identification system}

The identification system is based on a neural network. In this study, we developed two kinds of neural network structures. The first kind of structure (Structure 1 ) is made up of a mono block MultiLayer neural network with 3600 $(40 \times 90)$ inputs and 23 outputs. Only one output line is enabled (that is, 1 ) and all the others are disabled (i.e. -1 ) during the operation. The second type of structure (Structure 2) is made up of 23 network blocks. Each network block has $3600(40 \times 90)$ lines in parallel as inputs and two lines as outputs. The active output is $(1,-1)$ and inactive output is $(-1,1)$. During the operation, only one of the block 


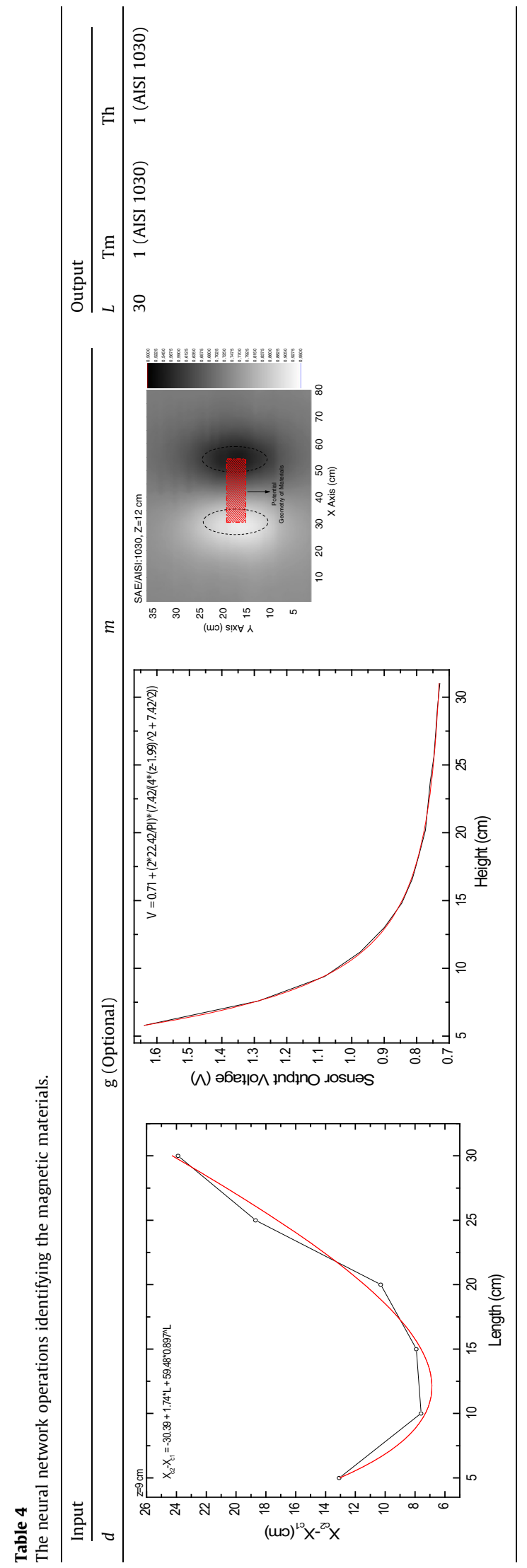

(a)

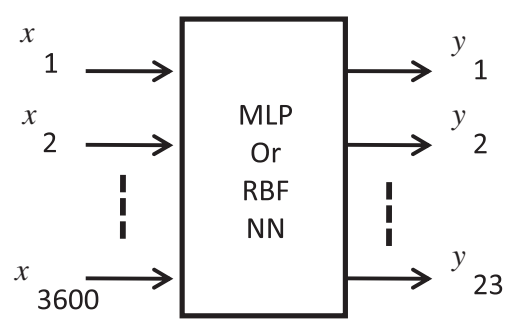

(b)

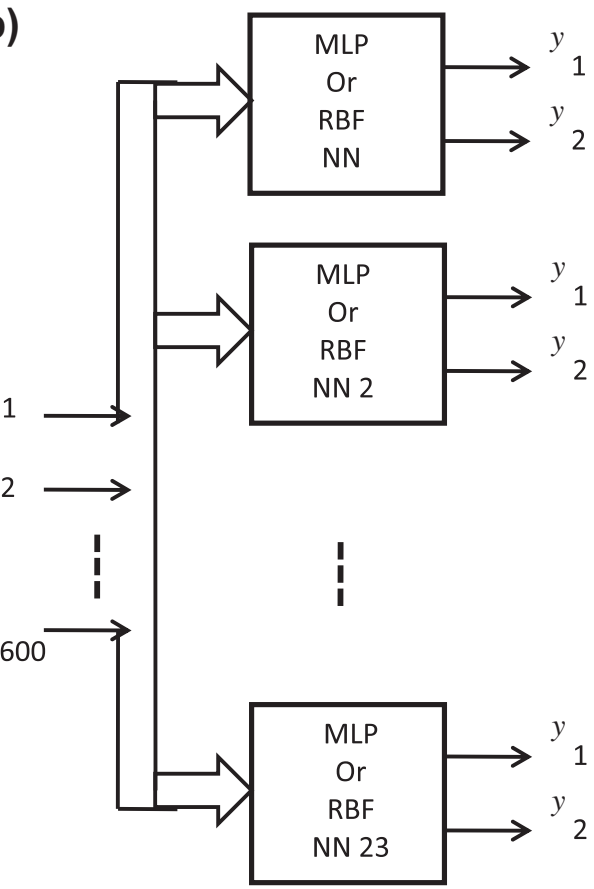

Fig. 5. The neural network structures used for the system implementation. (a) The first structure (Structure 1) for the Classifier A and B. (b) The second structure (Structure 2) for Classifier C and D.

produces $(1,-1)$ and all the others produce $(-1,1)$. These two structures are shown in Fig. 5. Based on the two structures and network types, four types of classifiers are implemented. They are called Classifier A, B, C, and $D$. The details of the classifiers are given in the next section, but as a first look, we can give a block diagram definition of them as follows. The Classifier A is a Structure 1 type network utilizing MultiLayer Perceptron (MLP). The Classifier B is also Structure 1 type neural network utilizing Radial Basis Function (RBF). The Classifier $\mathrm{C}$ is a Structure 2 type neural network utilizing MultiLayer Perceptron (MLP). The Classifier D is also Structure 2 type neural network utilizing Radial Basis Function (RBF).

A MultiLayer Perceptron (MLP) is a feedforward ANN (Artificial Neural Network) model based on supervised training. An MLP consists of a set of input units (the input layer), one or more sets of computation nodes (the hidden layers), and one set of computation/output nodes (the output layer). Connections are always made forward, on a layer-by-layer basis $[13,14]$. 


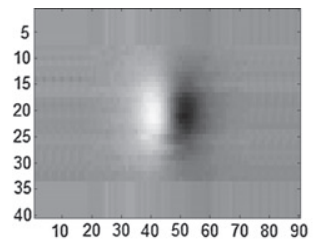

AISI1030A (Im-1)

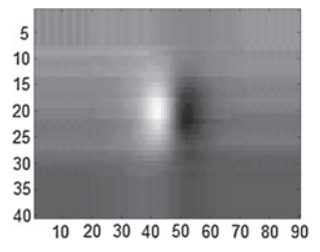

AISI1030E (Im-5)

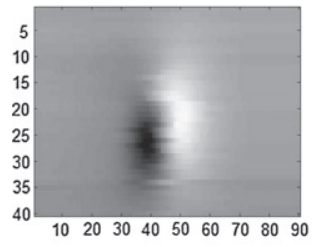

AISI1060B (Im-9)

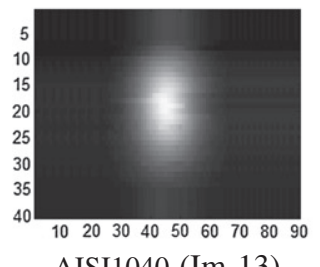

AISI1040 (Im-13)

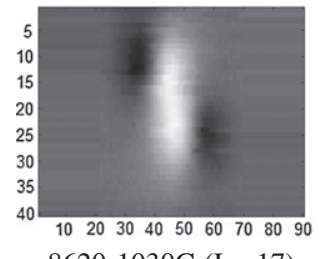

8620-1030G (Im-17)

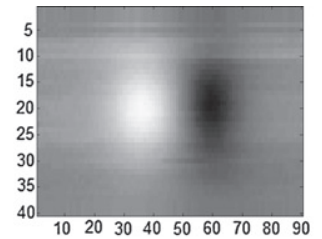

AISI1030B (Im-2)

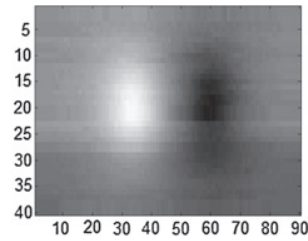

AISI1030F (Im-6)

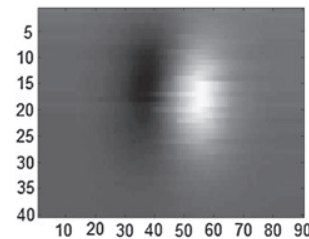

AISI1060C (Im-10)

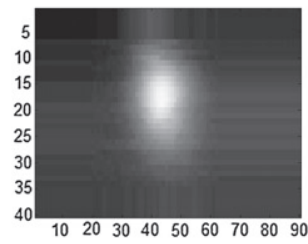

AISI1060S (Im-14)

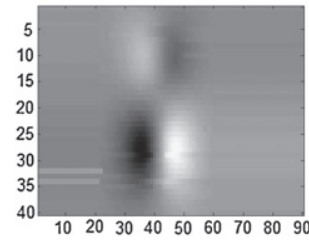

Two 1030C (Im-18)

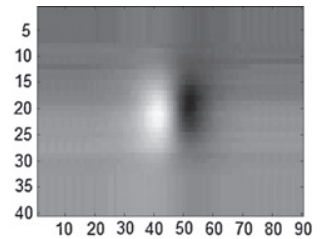

AISI1030C (Im-3)

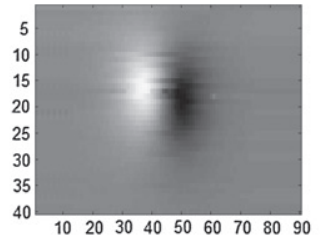

AISI1030G (Im-7)

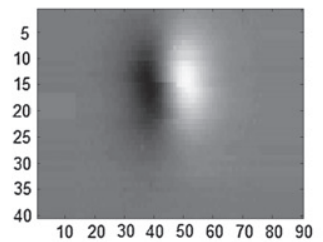

AISI1035A (Im-11)

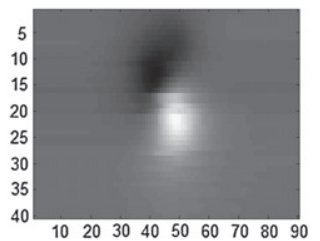

AISI4140 (Im-15)

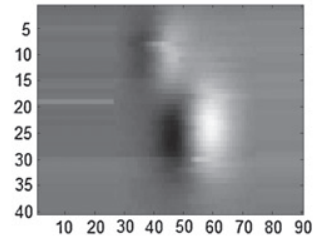

1035A-8620 (Im-19)

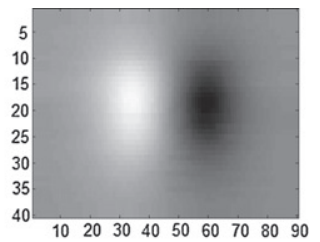

AISI1030D (Im-4)

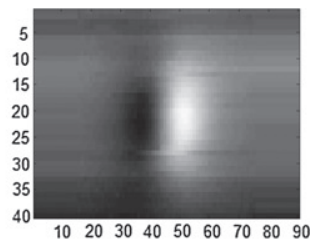

AISI1060A (Im-8)

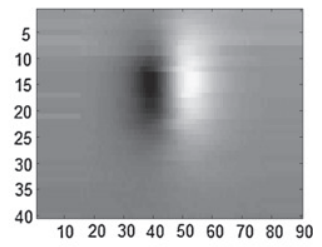

AISI1035B (Im-12)

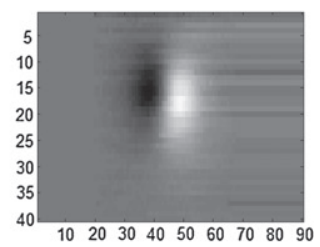

AISI8620 (Im-16)

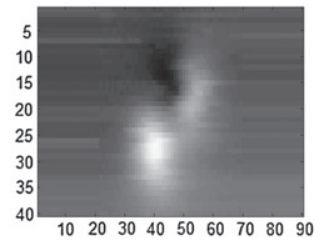

1060-8620 (Im-20)

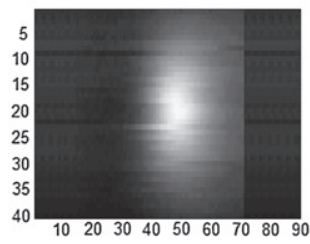

M2-AP-Mine (Im-21)

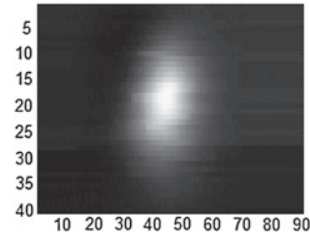

M16-AP-Mine (Im-22)

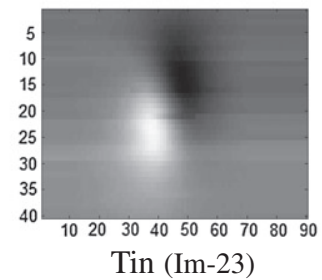

Tin (Im-23)

Fig. 6. Images of magnetic anomalies created by a couple of magnetic materials.

The RBF network is, like the MLP, a MultiLayer feedforward neural network. It has a single hidden layer while the MLP can have one or more [15]. In this work, the hidden layers in RBF networks use Gaussian density functions as their activation functions.

\section{Operation of the identification system and experimental results}

In Fig. 6, it can be seen 23 images with a dimension of $40 \times 90$ pixels. They are numerated as $I m-k$ for $k=1, \ldots$, 

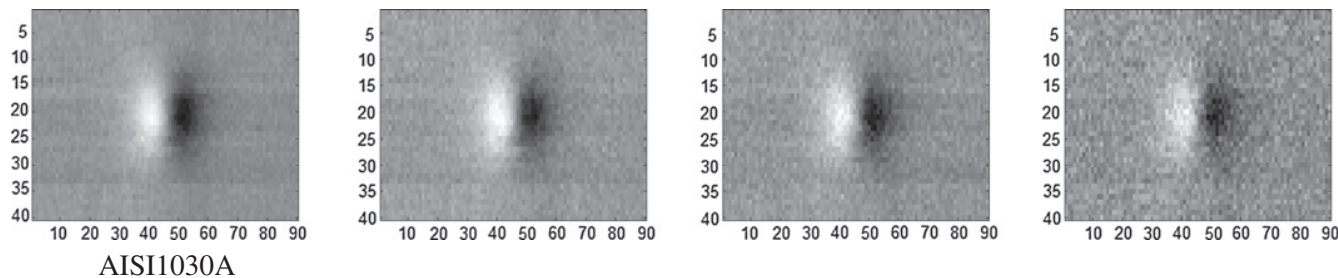

Fig. 7. Images with additive Gaussian white noise having zero means and the variances of $2 \times 10^{-6}, 5 \times 10^{-6}, 9 \times 10^{-6}$ ve $3 \times 10^{-5}$ from left to right respectively.

Table 5

Properties of Classifier A.

\begin{tabular}{|c|c|c|c|}
\hline Number of hidden layers & 3 & & \\
\hline \multirow[t]{3}{*}{ The type of activation functions and number of neurons used in hidden layers } & 1st hidden layer & Tangent-sigmoid & 30 \\
\hline & 2nd hidden layer & Tangent-sigmoid & 35 \\
\hline & 3rd hidden layer & Tangent-sigmoid & 35 \\
\hline Activation function used in output layer & Tangent-sigmoid & & \\
\hline Algorithm used in training & Scaled conjugate gradient backpropagation & & \\
\hline Training period & $266.86 \mathrm{~s}$ & & \\
\hline Mean square error (mse) value at the end of the training period & $9.26 \mathrm{e}-08$ & & \\
\hline
\end{tabular}

Table 6

Test results of classifier a having input images with an additive Gaussian white noise having zero mean and a variance of $2 \times 10^{-6}$.

\begin{tabular}{|c|c|c|c|c|c|}
\hline \multirow[t]{2}{*}{ Individual outputs of the Classifier A } & \multicolumn{5}{|c|}{ Image numbers of the inputs of the Classifier A with additive Gaussian white noise } \\
\hline & 1 & 6 & 11 & 18 & 21 \\
\hline 1 & 0.9995 & -1.0000 & -1.0000 & -1.0000 & -0.9998 \\
\hline 2 & -1.0000 & -0.9998 & -1.0000 & -1.0000 & -1.0000 \\
\hline 3 & -1.0000 & -1.0000 & -1.0000 & -0.9998 & -1.0000 \\
\hline 4 & -1.0000 & -0.9999 & -1.0000 & -1.0000 & -1.0000 \\
\hline 5 & -1.0000 & -1.0000 & -1.0000 & -0.9997 & -1.0000 \\
\hline 6 & -0.9995 & 0.9994 & -1.0000 & -1.0000 & -1.0000 \\
\hline 7 & -0.9997 & -0.9994 & -1.0000 & -1.0000 & -1.0000 \\
\hline 8 & -1.0000 & -1.0000 & -1.0000 & -0.9998 & -1.0000 \\
\hline 9 & -1.0000 & -1.0000 & -0.9998 & -0.9998 & -1.0000 \\
\hline 10 & -1.0000 & -1.0000 & -0.9998 & -1.0000 & -0.9999 \\
\hline 11 & -1.0000 & -1.0000 & 0.9993 & -0.9999 & -1.0000 \\
\hline 12 & -0.9999 & -1.0000 & -1.0000 & -1.0000 & -0.9995 \\
\hline 13 & -1.0000 & -0.9999 & -1.0000 & -1.0000 & -0.9999 \\
\hline 14 & -1.0000 & -1.0000 & -1.0000 & -1.0000 & -1.0000 \\
\hline 15 & -0.9999 & -1.0000 & -1.0000 & -1.0000 & -0.9999 \\
\hline 16 & -1.0000 & -0.9999 & -0.9994 & -1.0000 & -1.0000 \\
\hline 17 & -1.0000 & -1.0000 & -1.0000 & -0.9998 & -1.0000 \\
\hline 18 & -1.0000 & -1.0000 & -1.0000 & 0.9992 & -1.0000 \\
\hline 19 & -1.0000 & -1.0000 & -0.9998 & -0.9995 & -0.9998 \\
\hline 20 & -1.0000 & -1.0000 & -1.0000 & -0.9996 & -1.0000 \\
\hline 21 & -1.0000 & -0.9998 & -1.0000 & -1.0000 & 0.9990 \\
\hline 22 & -1.0000 & -1.0000 & -1.0000 & -0.9999 & -0.9990 \\
\hline 23 & -1.0000 & -0.9996 & -1.0000 & -1.0000 & -0.9999 \\
\hline
\end{tabular}

Table 7

Properties of the Classifier B.

\begin{tabular}{ll}
\hline Number of neurons in hidden layer & 67 \\
Spread parameter & 1 \\
Training period & $24.34 \mathrm{~s}$ \\
Mean square error (mse) value after training & $5.33 \mathrm{e}-09$ \\
\hline
\end{tabular}

23 for ease of handling. The height of the sensor is $z=10 \mathrm{~cm}$.

Before training operation, the Gaussian white noises with zero means and variances of $2 \times 10^{-6}, 5 \times 10^{-6}$,
$9 \times 10^{-6}$ and $3 \times 10^{-5}$ have been added to the images as shown in Fig. 7.

As it mentioned above, four types of classifiers (A, B, C, and $D$ ) based on artificial neural networks have been designed for the identification purpose of the images. During the training phases of the neural networks, the original images together with the images to which Gaussian white noise with zero mean and variances of $5 \times 10^{-6}$ ve $3 \times 10^{-5}$ added have been used. The trained neural nets have been tested with the images having an additive Gaussian white noise with zero mean and variances of $2 \times 10^{-6}$ 
Table 8

Test results of classifier b having input images with an additive gaussian white noise having zero mean and a variance of $9 \times 10^{-6}$.

\begin{tabular}{|c|c|c|c|c|c|}
\hline \multirow[t]{2}{*}{ Individual outputs of the Classifier B } & \multicolumn{5}{|c|}{ Image numbers of the inputs of the Classifier B with additive Gaussian white noise } \\
\hline & 5 & 7 & 13 & 19 & 22 \\
\hline 1 & -0.9982 & -1.0014 & -0.9998 & -0.9999 & -0.9999 \\
\hline 2 & -1.0037 & -1.0011 & -1.0007 & -1.0008 & -1.0002 \\
\hline 3 & -1.0127 & -1.0133 & -1.0129 & -1.0127 & -1.0118 \\
\hline 4 & -1.0000 & -1.0000 & -1.0000 & -1.0000 & -1.0000 \\
\hline 5 & 0.9501 & -0.9999 & -0.9998 & -0.9998 & -1.0003 \\
\hline 6 & -0.9996 & -0.9991 & -1.0000 & -1.0000 & -0.9995 \\
\hline 7 & -1.0001 & 0.9552 & -1.0000 & -1.0000 & -1.0000 \\
\hline 8 & -0.9996 & -1.0007 & -1.0005 & -1.0005 & -1.0019 \\
\hline 9 & -1.0003 & -0.9998 & -0.9999 & -0.9998 & -1.0001 \\
\hline 10 & -1.0001 & -1.0001 & -1.0001 & -1.0001 & -1.0001 \\
\hline 11 & -0.9976 & -0.9976 & -0.9973 & -0.9975 & -0.9972 \\
\hline 12 & -0.9998 & -0.9999 & -0.9999 & -1.0000 & -0.9999 \\
\hline 13 & -0.9997 & -0.9996 & 0.9547 & -0.9996 & -0.9997 \\
\hline 14 & -0.9879 & -0.9982 & -0.9989 & -0.9984 & -0.9973 \\
\hline 15 & -1.0000 & -1.0000 & -1.0000 & -1.0000 & -1.0000 \\
\hline 16 & -1.0047 & -1.0012 & -1.0019 & -1.0018 & -1.0018 \\
\hline 17 & -0.9420 & -0.9383 & -0.9404 & -0.9419 & -0.9453 \\
\hline 18 & -1.0036 & -1.0039 & -1.0038 & -1.0027 & -1.0034 \\
\hline 19 & -1.0001 & -1.0001 & -1.0002 & 0.9569 & -1.0001 \\
\hline 20 & -1.0000 & -1.0010 & -1.0009 & -1.0014 & -1.0008 \\
\hline 21 & -1.0000 & -1.0000 & -1.0000 & -1.0000 & -1.0000 \\
\hline 22 & -1.0006 & -1.0001 & -0.9977 & -1.0001 & 0.9594 \\
\hline 23 & -0.9999 & -1.0000 & -1.0000 & -1.0000 & -1.0000 \\
\hline
\end{tabular}

Table 9

Properties of the Classifier $\mathrm{C}$.

\begin{tabular}{|c|c|c|c|}
\hline $\begin{array}{l}\text { Sub classifiers forming the } \\
\text { Classifier C }\end{array}$ & $\begin{array}{l}\text { Number of neurons in hidden } \\
\text { layer }\end{array}$ & $\begin{array}{l}\text { Training } \\
\text { period }\end{array}$ & $\begin{array}{l}\text { Mean square error (mse) value at the end of the training } \\
\text { period }\end{array}$ \\
\hline 1 & 5 & $30.41 \mathrm{~s}$ & $2.39 \mathrm{e}-04$ \\
\hline 2 & 6 & $10.03 \mathrm{~s}$ & $6.26 \mathrm{e}-09$ \\
\hline 3 & 6 & $6.42 \mathrm{~s}$ & $7.47 e-08$ \\
\hline 4 & 6 & $3.89 \mathrm{~s}$ & $3.12 \mathrm{e}-08$ \\
\hline 5 & 6 & $9.61 \mathrm{~s}$ & $1.01 \mathrm{e}-07$ \\
\hline 6 & 6 & $14.26 \mathrm{~s}$ & $4.35 \mathrm{e}-08$ \\
\hline 7 & 6 & $4.14 \mathrm{~s}$ & $1.37 \mathrm{e}-07$ \\
\hline 8 & 6 & $15.70 \mathrm{~s}$ & $1.34 \mathrm{e}-08$ \\
\hline 9 & 6 & $5.23 \mathrm{~s}$ & $3.49 \mathrm{e}-08$ \\
\hline 10 & 6 & $6.52 \mathrm{~s}$ & $7.82 \mathrm{e}-08$ \\
\hline 11 & 6 & $3.73 \mathrm{~s}$ & $5.70 e-09$ \\
\hline 12 & 6 & $6.50 \mathrm{~s}$ & $1.36 \mathrm{e}-07$ \\
\hline 13 & 6 & $4.84 \mathrm{~s}$ & $7.67 \mathrm{e}-08$ \\
\hline 14 & 7 & $12.81 \mathrm{~s}$ & $7.97 e-08$ \\
\hline 15 & 6 & $4.65 \mathrm{~s}$ & $8.30 \mathrm{e}-08$ \\
\hline 16 & 7 & $20.89 \mathrm{~s}$ & $1.37 \mathrm{e}-07$ \\
\hline 17 & 6 & $21.91 \mathrm{~s}$ & $4.99 \mathrm{e}-08$ \\
\hline 18 & 6 & $5.98 \mathrm{~s}$ & $2.39 \mathrm{e}-08$ \\
\hline 19 & 6 & $4.06 \mathrm{~s}$ & $2.25 \mathrm{e}-08$ \\
\hline 20 & 6 & $14.74 \mathrm{~s}$ & $5.06 \mathrm{e}-08$ \\
\hline 21 & 6 & $7.75 \mathrm{~s}$ & $1.48 \mathrm{e}-08$ \\
\hline 22 & 8 & $12.16 \mathrm{~s}$ & $1.68 \mathrm{e}-07$ \\
\hline 23 & 6 & $5.70 \mathrm{~s}$ & $1.15 \mathrm{e}-08$ \\
\hline
\end{tabular}

ve $9 \times 10^{-6}$. This kind of testing ensures that the system can operate very well and achieve identification satisfactorily.

\subsection{Classifier A}

It is a neural network with $3600(40 \times 90)$ inputs and 23 outputs (Structure 1). It is a MultiLayer Perceptron (MLP). The structure and training details of the classifier are given in Table 5.
The results of some of the outputs during the transformation phase after the training where the inputs to the Classifier A are images with additive Gaussian white noise having zero mean and a variance of $2 \times 10^{-6}$ are given in Table 6. Similarly, the results of some of the outputs during the transformation phase after the training where the inputs to the Classifier A are images with additive Gaussian white noise having zero mean and a variance of $9 \times 10^{-6}$ are obtained. $100 \%$ accuracy have been obtained in both testings. The accuracy is calculated as 
Table 10

Test results of classifier c having input images with an additive gaussian white noise having zero mean and a variance of $2 \times 10^{-6}$.

\begin{tabular}{|c|c|c|c|c|c|c|}
\hline \multirow[t]{2}{*}{ Sub classifiers } & \multirow{2}{*}{$\begin{array}{l}\text { Outputs of } \\
\text { sub classifiers }\end{array}$} & \multicolumn{5}{|c|}{ Input image numbers with additive Gaussian white noise } \\
\hline & & 4 & 9 & 14 & 17 & 23 \\
\hline \multirow[t]{2}{*}{1} & 1 & -0.9995 & -0.9998 & -0.9997 & -0.9941 & -0.9994 \\
\hline & 2 & 0.9995 & 0.9998 & 0.9997 & 0.9933 & 0.9993 \\
\hline \multirow[t]{2}{*}{2} & 1 & -0.9999 & -0.9997 & -0.9999 & -0.9999 & -0.9999 \\
\hline & 2 & 1.0000 & 1.0000 & 1.0000 & 1.0000 & 1.0000 \\
\hline \multirow[t]{2}{*}{3} & 1 & -0.9996 & -0.9999 & -0.9998 & -0.9991 & -0.9992 \\
\hline & 2 & 0.9998 & 1.0000 & 1.0000 & 0.9999 & 0.9998 \\
\hline \multirow[t]{2}{*}{4} & 1 & 0.9990 & -1.0000 & -1.0000 & -1.0000 & -1.0000 \\
\hline & 2 & -0.9995 & 0.9999 & 0.9999 & 0.9999 & 0.9999 \\
\hline \multirow[t]{2}{*}{5} & 1 & -1.0000 & -1.0000 & -0.9999 & -0.9999 & -1.0000 \\
\hline & 2 & 0.9996 & 0.9996 & 0.9996 & 0.9996 & 0.9997 \\
\hline \multirow[t]{2}{*}{6} & 1 & -0.9992 & -0.9999 & -1.0000 & -1.0000 & -1.0000 \\
\hline & 2 & 1.0000 & 1.0000 & 1.0000 & 1.0000 & 1.0000 \\
\hline \multirow[t]{2}{*}{7} & 1 & -0.9989 & -0.9999 & -0.9999 & -0.9999 & -0.9999 \\
\hline & 2 & 0.9990 & 0.9999 & 0.9999 & 0.9999 & 0.9998 \\
\hline \multirow[t]{2}{*}{8} & 1 & -1.0000 & -1.0000 & -1.0000 & -1.0000 & -0.9997 \\
\hline & 2 & 1.0000 & 1.0000 & 1.0000 & 1.0000 & 0.9999 \\
\hline \multirow[t]{2}{*}{9} & 1 & -1.0000 & 0.9993 & -1.0000 & -1.0000 & -1.0000 \\
\hline & 2 & 0.9999 & -0.9992 & 0.9999 & 0.9999 & 0.9999 \\
\hline \multirow[t]{2}{*}{10} & 1 & -1.0000 & -0.9999 & -1.0000 & -1.0000 & -1.0000 \\
\hline & 2 & 1.0000 & 1.0000 & 1.0000 & 1.0000 & 1.0000 \\
\hline \multirow[t]{2}{*}{11} & 1 & -1.0000 & -1.0000 & -1.0000 & -1.0000 & -1.0000 \\
\hline & 2 & 1.0000 & 1.0000 & 1.0000 & 1.0000 & 1.0000 \\
\hline \multirow[t]{2}{*}{12} & 1 & -0.9995 & -0.9995 & -0.9995 & -0.9995 & -0.9995 \\
\hline & 2 & 1.0000 & 1.0000 & 1.0000 & 1.0000 & 1.0000 \\
\hline \multirow[t]{2}{*}{13} & 1 & -0.9996 & -0.9996 & -0.9996 & -0.9996 & -0.9996 \\
\hline & 2 & 1.0000 & 1.0000 & 1.0000 & 1.0000 & 1.0000 \\
\hline \multirow[t]{2}{*}{14} & 1 & -0.9999 & -0.9998 & 0.9989 & -0.9998 & -1.0000 \\
\hline & 2 & 0.9999 & 0.9999 & -0.9992 & 0.9999 & 1.0000 \\
\hline \multirow[t]{2}{*}{15} & 1 & -0.9996 & -0.9998 & -0.9998 & -0.9998 & -0.9998 \\
\hline & 2 & 1.0000 & 0.9999 & 0.9999 & 0.9999 & 0.9999 \\
\hline \multirow[t]{2}{*}{16} & 1 & -0.9995 & -1.0000 & -1.0000 & -0.9995 & -0.9996 \\
\hline & 2 & 0.9997 & 1.0000 & 1.0000 & 0.9997 & 0.9998 \\
\hline \multirow[t]{2}{*}{17} & 1 & -0.9999 & -1.0000 & -1.0000 & 0.9992 & -1.0000 \\
\hline & 2 & 1.0000 & 1.0000 & 1.0000 & -0.9988 & 1.0000 \\
\hline \multirow[t]{2}{*}{18} & 1 & -0.9999 & -0.9998 & -1.0000 & -0.9999 & -1.0000 \\
\hline & 2 & 1.0000 & 0.9997 & 0.9999 & 0.9999 & 0.9999 \\
\hline \multirow[t]{2}{*}{19} & 1 & -1.0000 & -1.0000 & -1.0000 & -1.0000 & -1.0000 \\
\hline & 2 & 1.0000 & 0.9999 & 1.0000 & 1.0000 & 1.0000 \\
\hline 20 & 1 & -1.0000 & -1.0000 & -1.0000 & -1.0000 & -1.0000 \\
\hline & 2 & 1.0000 & 1.0000 & 1.0000 & 1.0000 & 1.0000 \\
\hline 21 & 1 & -1.0000 & -0.9999 & -1.0000 & -1.0000 & -1.0000 \\
\hline & 2 & 1.0000 & 0.9999 & 1.0000 & 1.0000 & 1.0000 \\
\hline 22 & 1 & -0.9998 & -1.0000 & -0.9996 & -0.9998 & -0.9998 \\
\hline & 2 & 0.9997 & 1.0000 & 0.9995 & 0.9997 & 0.9997 \\
\hline 23 & 1 & -0.9998 & -1.0000 & -1.0000 & -1.0000 & 1.0000 \\
\hline & 2 & 0.9999 & 1.0000 & 1.0000 & 1.0000 & -0.9993 \\
\hline
\end{tabular}

Accuracy $=$ (Number of correctly identified $\times$ images/Number of images) $\times 100$

\subsection{Classifier B}

It is a neural network with $3600(40 \times 90)$ inputs and 23 outputs (Structure 1). It is a Radial Basis Function (RBF). The structure and training details of the classifier are given in Table 7. In this network, the number of neurons in hidden layer is increased from one to 67 one-by-one until obtaining the best result.

The training period of Classifier B is shorter in comparison with the Classifier $A$. The value of the mean square error of the Classifier B is lower than the Classifier A as well. The results of some of the outputs during the transformation phase after the training where the inputs to the Classifier $B$ are images with additive Gaussian white noise having zero mean and a variance of $2 \times 10^{-6}$ are obtained. Similarly, the results of some of the outputs during the transformation phase after the training where the inputs to the Classifier B are images with additive Gaussian white noise having zero mean and a variance of $9 \times 10^{-6}$ are given in Table $8.100 \%$ accuracy have been obtained in both testings.

\subsection{Classifier $C$}

It is a neural network which is composed of 23 modules of MultiLayer Perceptron (MLP). Each module has 3600 $(40 \times 90)$ inputs, one hidden layer and two outputs (Structure 2). A tangent-sigmoid activation function is used at the outputs of both hidden layer neurons and output layer 
Table 11

Properties of the Classifier D.

\begin{tabular}{clll}
\hline $\begin{array}{l}\text { Sub classifiers } \\
\text { forming the } \\
\text { Classifier D }\end{array}$ & $\begin{array}{l}\text { Number of } \\
\text { neurons in } \\
\text { hidden layer }\end{array}$ & $\begin{array}{l}\text { Training } \\
\text { period }\end{array}$ & $\begin{array}{l}\text { Mean square error } \\
\text { (mse) value at the end } \\
\text { of the training period }\end{array}$ \\
\hline 1 & 30 & $4.67 \mathrm{~s}$ & $7.40 \mathrm{e}-05$ \\
2 & 30 & $5.32 \mathrm{~s}$ & $2.00 \mathrm{e}-04$ \\
3 & 30 & $5.38 \mathrm{~s}$ & $6.20 \mathrm{e}-06$ \\
4 & 30 & $4.61 \mathrm{~s}$ & $4.29 \mathrm{e}-06$ \\
5 & 30 & $4.89 \mathrm{~s}$ & $3.15 \mathrm{e}-05$ \\
6 & 30 & $4.69 \mathrm{~s}$ & $6.41 \mathrm{e}-05$ \\
7 & 30 & $4.81 \mathrm{~s}$ & $9.10 \mathrm{e}-06$ \\
8 & 30 & $4.61 \mathrm{~s}$ & $4.31 \mathrm{e}-04$ \\
9 & 30 & $5.37 \mathrm{~s}$ & $8.49 \mathrm{e}-05$ \\
10 & 30 & $4.66 \mathrm{~s}$ & $4.43 \mathrm{e}-05$ \\
11 & 30 & $4.62 \mathrm{~s}$ & $5.02 \mathrm{e}-04$ \\
12 & 30 & $4.66 \mathrm{~s}$ & $2.59 \mathrm{e}-05$ \\
13 & 30 & $4.96 \mathrm{~s}$ & $4.62 \mathrm{e}-07$ \\
14 & 30 & 4.96 & $7.26 \mathrm{e}-05$ \\
15 & 20 & 4.07 & $1.94 \mathrm{e}-04$ \\
16 & 30 & 5.00 & $4.67 \mathrm{e}-05$ \\
17 & 30 & 4.75 & $7.67 \mathrm{e}-05$ \\
18 & 30 & 4.94 & $6.23 \mathrm{e}-07$ \\
19 & 30 & 4.76 & $1.07 \mathrm{e}-06$ \\
20 & 30 & 5.02 & $7.71 \mathrm{e}-05$ \\
21 & 30 & 4.76 & $4.78 \mathrm{e}-06$ \\
22 & 30 & 4.76 & $3.02 \mathrm{e}-04$ \\
23 & 30 & 4.84 & $7.00 \mathrm{e}-06$ \\
\hline & & & \\
\hline & 30 & & \\
\hline & 30 & &
\end{tabular}

neurons. The training algorithm used is the scaled conjugate gradient backpropagation. The structure and training details of the classifier are given in Table 9.

The average training period of Classifier $C$ is $10.08 \mathrm{~s}$ and the average mean square error value at the end of the training period of Classifier $\mathrm{C}$ is $1.05 \mathrm{e}-05$.

During the testing phase, the same image is applied to 23 sub modules in parallel and the outputs are obtained simultaneously. The results of some of the outputs during the transformation phase after the training where the inputs to the Classifier $C$ are images with additive Gaussian white noise having zero mean and a variance of $2 \times 10^{-6}$ are given in Table 10. Similarly, the results of some of the outputs during the transformation phase after the training where the inputs to the Classifier $C$ are images with additive Gaussian white noise having zero mean and a variance of $9 \times 10^{-6}$ are obtained. $100 \%$ accuracy have been obtained in both testings.

\subsection{Classifier $D$}

It is a neural network which is composed of 23 modules of Radial Basis Function (RBF) Networks. Each module has $3600(40 \times 90)$ inputs and two outputs (Structure 2$)$. In this network, the number of neurons in hidden layer is increased from one to 30 one-by-one until obtaining the best result. The spread parameter is taken as 1 . The structure and training details of the classifier are given in Table 11.

The average training period of Classifier D is $4.83 \mathrm{~s}$ and the average mean square error value at the end of the training period of Classifier D is $9.83 e-05$.

During the testing phase, the same image is applied to 23 sub modules in parallel and the outputs are obtained
Table 12

Test results of classifier $\mathrm{d}$ having input images with an additive gaussian white noise having zero mean and a variance of $9 \times 10^{-6}$.

\begin{tabular}{|c|c|c|c|c|c|c|}
\hline \multirow[t]{2}{*}{$\begin{array}{l}\text { Sub } \\
\text { classifiers }\end{array}$} & \multirow{2}{*}{$\begin{array}{l}\text { Outputs } \\
\text { of sub } \\
\text { classifiers }\end{array}$} & \multicolumn{5}{|c|}{$\begin{array}{l}\text { Input image numbers with additive Gaussian } \\
\text { white noise }\end{array}$} \\
\hline & & 3 & 8 & 13 & 20 & 22 \\
\hline \multirow[t]{2}{*}{1} & 1 & -0.9992 & -1.0031 & -1.0002 & -0.9994 & -1.0037 \\
\hline & 2 & 0.9992 & 1.0031 & 1.0002 & 0.9994 & 1.0037 \\
\hline \multirow[t]{2}{*}{2} & 1 & -0.9834 & -1.0403 & -1.0022 & -1.0048 & -0.9902 \\
\hline & 2 & 0.9834 & 1.0403 & 1.0022 & 1.0048 & 0.9902 \\
\hline \multirow[t]{2}{*}{3} & 1 & 0.9669 & -0.9992 & -0.9998 & -0.9970 & -0.9992 \\
\hline & 2 & -0.9669 & 0.9992 & 0.9998 & 0.9970 & 0.9992 \\
\hline \multirow[t]{2}{*}{4} & 1 & -1.0000 & -1.0000 & -1.0000 & -1.0000 & -0.9999 \\
\hline & 2 & 1.0000 & 1.0000 & 1.0000 & 1.0000 & 0.9999 \\
\hline \multirow[t]{2}{*}{5} & 1 & -1.0006 & -0.9942 & -1.0002 & -0.9996 & -1.0024 \\
\hline & 2 & 1.0006 & 0.9942 & 1.0002 & 0.9996 & 1.0024 \\
\hline \multirow[t]{2}{*}{6} & 1 & -1.0001 & -0.9938 & -1.0001 & -1.0002 & -0.9946 \\
\hline & 2 & 1.0001 & 0.9938 & 1.0001 & 1.0002 & 0.9946 \\
\hline \multirow[t]{2}{*}{7} & 1 & -1.0000 & -0.9976 & -1.0002 & -1.0000 & -0.9997 \\
\hline & 2 & 1.0000 & 0.9976 & 1.0002 & 1.0000 & 0.9997 \\
\hline \multirow[t]{2}{*}{8} & 1 & -1.0029 & 0.9749 & -1.0031 & -0.9918 & -0.9771 \\
\hline & 2 & 1.0029 & -0.9749 & 1.0031 & 0.9918 & 0.9771 \\
\hline \multirow[t]{2}{*}{9} & 1 & -0.9988 & -0.9637 & -1.0004 & -0.9988 & -0.9976 \\
\hline & 2 & 0.9988 & 0.9637 & 1.0004 & 0.9988 & 0.9976 \\
\hline \multirow[t]{2}{*}{10} & 1 & -1.0001 & -0.9882 & -1.0004 & -0.9996 & -0.9973 \\
\hline & 2 & 1.0001 & 0.9882 & 1.0004 & 0.9996 & 0.9973 \\
\hline \multirow[t]{2}{*}{11} & 1 & -1.0020 & -0.9970 & -1.0044 & -1.0044 & -0.9849 \\
\hline & 2 & 1.0020 & 0.9970 & 1.0044 & 1.0044 & 0.9849 \\
\hline \multirow[t]{2}{*}{12} & 1 & -1.0003 & -0.9959 & -1.0002 & -0.9990 & -0.9971 \\
\hline & 2 & 1.0003 & 0.9959 & 1.0002 & 0.9990 & 0.9971 \\
\hline \multirow[t]{2}{*}{13} & 1 & -1.0000 & -1.0000 & 0.9550 & -0.9997 & -0.9950 \\
\hline & 2 & 1.0000 & 1.0000 & -0.9550 & 0.9997 & 0.9950 \\
\hline \multirow[t]{2}{*}{14} & 1 & -1.0003 & -0.9812 & -1.0001 & -0.9873 & -0.9919 \\
\hline & 2 & 1.0003 & 0.9812 & 1.0001 & 0.9873 & 0.9919 \\
\hline \multirow[t]{2}{*}{15} & 1 & -1.0000 & -1.0000 & -1.0000 & -1.0000 & -1.0000 \\
\hline & 2 & 1.0000 & 1.0000 & 1.0000 & 1.0000 & 1.0000 \\
\hline \multirow[t]{2}{*}{16} & 1 & -1.0003 & -0.9920 & -1.0002 & -1.0052 & -1.0199 \\
\hline & 2 & 1.0003 & 0.9920 & 1.0002 & 1.0052 & 1.0199 \\
\hline \multirow[t]{2}{*}{17} & 1 & -0.9974 & -0.9950 & -0.9979 & -0.9995 & -0.9983 \\
\hline & 2 & 0.9974 & 0.9950 & 0.9979 & 0.9995 & 0.9983 \\
\hline \multirow[t]{2}{*}{18} & 1 & -0.9995 & -1.0000 & -1.0001 & -1.0007 & -1.0001 \\
\hline & 2 & 0.9995 & 1.0000 & 1.0001 & 1.0007 & 1.0001 \\
\hline \multirow[t]{2}{*}{19} & 1 & -1.0002 & -0.9999 & -0.9999 & -0.9995 & -0.9997 \\
\hline & 2 & 1.0002 & 0.9999 & 0.9999 & 0.9995 & 0.9997 \\
\hline 20 & 1 & -1.0007 & -0.9987 & -1.0004 & 0.9696 & -1.0014 \\
\hline & 2 & 1.0007 & 0.9987 & 1.0004 & -0.9696 & 1.0014 \\
\hline 21 & 1 & -0.9999 & -1.0000 & -1.0000 & -0.9999 & -0.9998 \\
\hline & 2 & 0.9999 & 1.0000 & 1.0000 & 0.9999 & 0.9998 \\
\hline 22 & 1 & -0.9818 & -0.9944 & -0.9847 & -0.9875 & 0.9689 \\
\hline & 2 & 0.9818 & 0.9944 & 0.9847 & 0.9875 & -0.9689 \\
\hline 23 & 1 & -0.9998 & -0.9991 & -1.0000 & -0.9995 & -1.0004 \\
\hline & 2 & 0.9998 & 0.9991 & 1.0000 & 0.9995 & 1.0004 \\
\hline
\end{tabular}

simultaneously. The results of some of the outputs during the transformation phase after the training where the inputs to the Classifier D are images with additive Gaussian white noise having zero mean and a variance of $9 \times 10^{-6}$ are given in Table 12. Similarly, the results of some of the outputs during the transformation phase after the training where the inputs to the Classifier D are images with additive Gaussian white noise having zero mean and a variance of $2 \times 10^{-6}$ are obtained. $100 \%$ accuracy have been obtained in both testings.

The training periods of Classifier $C$ and Classifier D are shorter than the training periods of Classifier A and Classifier B. However, Classifier B has the lowest mean square 
error value at the end of training period in comparison with the other classifiers.

\section{Conclusion}

In the study on materials with magnetic characteristics, we understand that the identification of magnetic materials can be achieved successfully by assessing the magnetic anomalies which occurred at the vertical component of the Earth magnetic field. During the studies all of the measurements related to the anomalies have been done by a KMZ51 MR sensor. In the previous work [1], first, the appropriate heights of sensor are determined for magnetic materials having various chemical contents. Then, how the identification of materials with their lengths, diameters, and upper surface images can be achieved is explained by a concrete example. The types of magnetic materials used in industry can be created by changing the chemical content of the materials based on their application areas. For example, carbon increases the hardness of a steel, but sulfur and phosphorous may make it fragile. Hence, what type of magnetic material which has been used for a product is previously known. Therefore, identification of a magnetic characteristic is not our business. This is nota parameter that we have to determine. However, a necessary condition is that the measurement system has to be calibrated previously for each kind of materials. Also, it is necessary to create a database storing the graphics shown in Fig. 4 for each type of material. Then, the length or diameter of the material can be determined by this measurement system. In this paper, we tried to identify a magnetic material by means of an intelligent system, that is, a neural network autonomously. A database holding 23 types of magnetic materials with various chemical contents is created. They are used to train the neural network system. The capacity of the database can easily be extended with new kinds of materials by use of the measurement system developed here. There are some restrictions on this paper. It is clear that the change in the Earth magnetic field in different regions and the availability of some sources of magnetic field other than the Earth magnetic field may affect the sensor output voltage. In such an environment, it is more difficult to determine the dimensions of a material. Furthermore, it must be noted that the method proposed for the determination of dimension depends also on the value of the homogeneous Earth magnetic field. For example, while the strength of the field is $4.4 \times 10^{-5}$ Tin the region where the measurements took place, it may be different in other locations. Therefore, it is essential to calibrate the measurement system in the region where it has to be used. However, in practice, it is desired that an autonomous identification system must be robust enough and does not necessitate an accurate calibration. Therefore, we utilized a neural network system that can easily elimi- nate these kinds of requirements. The resolution of the ADC is 12 bit. It is more than enough for obtaining the images of the magnetic anomalies. The main advantage of this measurement system is that it utilizes a sensor measuring directly the Earth's magnetic field. It gives the user a more accurate system with less power consumption. This is an innovative system that is used here, and it differs from the similar systems used in industry today. The system proposed here is able to give directly the length or the diameter of a material from the curves. The neural network that we use is able to decide whether the material is cylindrical or prismatic by training fitted curves automatically based on the characteristic variations and determining the variables $d=x_{c 2}-x_{c 1}$ or $W$ on these curves. The last but not least, the system proposed here can easily be used in buried mine detection and identification purposes. Actually, we have already given two kinds of buried mines such as anti-personal mine and anti-tank mine to be identified easily as seen in 21 st and 22 nd samples trained.

\section{References}

[1] Y. Ege, M.G. Gokhan, O. Kalender, S. Nazlibilek, Numerical analysis for remote identification of materials with magnetic characteristics, in: IEEE Trans. on Instrumentation and Measurement, vol. 60, No.9, September 2011.

[2] J.E. McFee, Y. Das, Locating and identifying compact ferrous objects, IEEE Trans. Geosci. Remote Sens. 28 (2) (1990) 182-193 (March).

[3] Y. Ege, O. Kalender, S. Nazlibilek, Direction finding of moving ferromagnetic objects inside water by magnetic anomaly, Sens. Actuators A, Phys. 147 (1) (2008) 52-59 (September).

[4] M.H. Kang, B.W. Choi, K.C. Koh, J.H. Lees, G.T. Park, Experimental study of a vehicle detector with an AMR sensor, Sens. Actuators A, Phys. 118 (2) (2005) 278-284. February.

[5] M. Woloszyn, Detection of ferromagnetic objects in local anomaly of the Baltic Sea, Polish Maritime Res. 15 (2) (2008) 77-82 (April).

[6] E. Galanzha, E.V. Shashkov, T. Kelly, J.-W. Kim, L. Yang, V. Zharov, In vivo magnetic enrichment and multiplex photoacoustic detection of circulating tumour cells, Nat. Nanotechnol. 4 (12) (2009) 855-860 (November).

[7] S. Piro, A. Samir, L. Versino, Position and spatial orientation of magnetic bodies from archeological magnetic surveys, Annali Di Geofisica 41 (3) (1998) 343-358 (August).

[8] T.R. Clem, Sensor technologies for hunting buried sea mines, in: Proc. MTS/IEEE Conf. OCEANS, 2002, vol. 1, pp. 452-460.

[9] T. El Tobelyl, A. Salem, Position detection of unexploded ordnance from airborne magnetic anomaly data using 3-D self organized feature map, in: Proc. 5th IEEE Int. Symp. Signal Process. Inf. Technol., Athens, Greece, vol. 1/2, pp. 322-327, 2005.

[10] E. Demircioglu, M.H. Sazli, Behavioral modeling of a C-band ring hybrid coupler using artificial neural networks, Radioengineering 19 (4) (2010) 645-652.

[11] M.H. Sazli, I. Can, Neural network implementation of the BCJR algorithm, Digital Signal Process. 17 (1) (2007) 353-359.

[12] KMZ51 Magnetic Field Sensor, Data Sheet, 2000.

[13] S. Haykin, Neural Networks and Learning Machines, Pearson Education Inc., New Jersey, 2009.

[14] J.F. Mas, J.J. Flores, The application of artificial neural networks to the analysis of remotely sensed data, Int. J. Remote Sens. 29 (3) (2008) 617-663 (February).

[15] G.M. Foody, Supervised image classification by MLP and RBF neural networks with and without an exhaustively defined set of classes, Int. J. Remote Sens. 25 (15) (2004) 3091-3104 (August). 